\title{
The isolation of spatial patterning modes in a mathematical model of juxtacrine cell signalling
}

\author{
O’Dea, R.D.* \& King, J.R. \\ Centre for Mathematical Medicine and Biology, \\ School of Mathematical Sciences, \\ University of Nottingham, University Park, \\ Nottingham, NG7 2RD, UK
}

June 11, 2015

\begin{abstract}
Juxtacrine signalling mechanisms are known to be crucial in tissue and organ development, leading to spatial patterns in gene expression. We investigate the patterning behaviour of a discrete model of juxtacrine cell signalling due to Owen \& Sherratt (Math. Biosci., 1998, 153(2):125-150) in which ligand molecules, unoccupied receptors and bound ligand-receptor complexes are modelled. Feedback between the ligand and receptor production and the level of bound receptors is incorporated. By isolating two parameters associated with the feedback strength and employing numerical simulation, linear stability and bifurcation analysis, the pattern-forming behaviour of the model is analysed under regimes corresponding to lateral inhibition and induction. Linear analysis of this model fails to capture the patterning behaviour exhibited in numerical simulations. Via bifurcation analysis we show that, since the majority of periodic patterns fold subcritically from the homogeneous steady state, a wide variety of stable patterns exists at a given parameter set, providing an explanation for this failure. The dominant pattern is isolated via numerical simulation. Additionally, by sampling patterns of non-integer wavelength on a discrete mesh, we highlight a disparity between the continuous and discrete representations of signalling mechanisms: in the continuous case, patterns of arbitrary wavelength are possible, while sampling such patterns on a discrete mesh leads to longer wavelength harmonics being selected where the wavelength is rational; in the irrational case, the resulting aperiodic patterns exhibit 'local periodicity', being constructed from distorted stable shorter-wavelength patterns. This feature is consistent with experimentally observed patterns, which typically display approximate short-range periodicity with defects.
\end{abstract}

\section{Introduction}

Cell-to-cell communication plays a key role in the development of multicellular organisms (Hartenstein \& Posakony, 1990; Keener \& Sneyd, 1998; Mitsiadis et al.,

\footnotetext{
${ }^{*}$ Corresponding author email: reuben.odea@ntu.ac.uk; Current address: School of Science and Technology, Nottingham Trent University, Nottingham, NG11 8NS, UK
} 
1999), leading to spatial patterns in gene expression that impact upon cell differentiation, the determination of cell fate and, ultimately, the development of tissues and organs. The activity of cell signalling molecules is typically divided into four distinct groups, termed autocrine, paracrine, endocrine and juxtacrine. The first three refer, respectively, to scenarios in which a cell produces a signalling molecule which is free to move within the tissue and acts (i) on the same cell, (ii) on a group of neighbouring cells (typically via diffusion) and (iii) on all cells within a tissue (as is the case with hormones). In contrast, juxtacrine signalling refers to the case where the signalling molecule is anchored in the cell membrane and acts only on neighbouring cells. The efficacy of such a mechanism is accordingly limited to closely packed structures such as epithelia.

Due to their importance in tissue development, cell signalling mechanisms have been the subject of a large number of theoretical studies. In this paper, we concentrate on a discrete mathematical model for juxtacrine signalling; a review of alternative cell signalling models is therefore omitted (see, e.g., Pribyl et al. (2003) and Muratov \& Shvartsman (2004) for discrete analyses of autocrine signalling).

The study of Turing (1952), which showed that reaction and diffusion of chemicals can produce spatial patterning in chemical concentration that consequently determines cell fate, has inspired many authors to employ continuum reaction-diffusion models to study patterning in biological systems (though we note that Turing (1952) exploited both discrete and continuous formulations) - see, for instance, Wolpert (1969), Painter et al. (1999) and Lander et al. (2002). Such models are posed in terms of partial differential equations and as such analytic or asymptotic solutions may sometimes be obtained, as well as numerical-simulation approaches undertaken. However, the above continuum studies are inappropriate for small numbers of cells or for the study of fine-grained patterns in which variation takes place over few cell diameters. For these reasons discrete models are often used, taking the form of systems of ordinary differential equations (ODEs) defined at discrete points in space, representing individual cells. The ease with which short range patterns and individual cell behaviour or movement may be captured in such models has led them to be widely exploited to model many aspects of cell behaviour. The analysis of discrete models can rely heavily on numerical simulation, leading to an upper limit on the size of the problem that can be investigated; additionally, analytical results may be difficult or impossible to obtain from a discrete model considering realistic numbers of cells. Some authors have therefore attempted to derive continuum models based upon an underlying discrete system. Examples include Turner et al. (2004), who showed that, in the continuum limit, a Potts model of cell movement may be represented by a diffusion equation, and O'Dea \& King (2011a,b) in which multiscale continuum models capable of describing certain short-range patterns in square and hexagonal cells brought about by a simple juxtacrine signalling were formulated. We note that the techniques presented in these studies may be applied to the more complex model analysed herein; the calculation is summarised within the Appendix.

A mathematical model for juxtacrine signalling was first considered by Collier et al. (1996), concentrating on the activity of a transmembrane protein, Delta, and its receptor, Notch. Lateral inhibition, a negative feedback mechanism by which a cell adopting a particular fate inhibits neighbouring cells from doing likewise (Goriely et al., 1991; Heitzler \& Simpson, 1991; Appel et al., 2001), was considered and shown to generate fine-grained patterns robustly. This mechanism is a fundamental 
cell fate control mechanism (Mitsiadis et al., 1999), creating fine-grained patterns in developing tissue which determine subsequent cell development.

Plahte (2001) provided a comprehensive analysis of pattern formation in continuous and discrete systems, with application to the model of Collier et al. (1996) and concluded that linear analysis may not provide sufficient information to explain patterning behaviour. Plahte \& Øyehaug (2007) suggests that in one spatial dimension, travelling waves invading an unstable homogeneous state may only generate periodtwo patterns, providing an explanation for the robustness of fine-grained patterning in this system.

Owen \& Sherratt (1998) analysed a more complicated juxtacrine signalling model, considering the numbers of ligand and free and bound receptors. Lateral induction (positive feedback between ligand-receptor binding and subsequent ligand production) was accommodated and the range over which juxtacrine signals may be transmitted was studied. Lateral induction is well-documented for a number of ligand-receptor interactions, including, for instance, the binding of cAMP to Dictyostelium cells (Owen \& Sherratt (1998) and references therein). In a subsequent paper, Owen et al. (1999) employed both a discrete (identical to that presented in Owen \& Sherratt (1998)) and a continuous formulation to investigate the propagation of signals in a juxtacrine signalling system, demonstrating that arbitrarily large signal half-lives are achievable and showing good agreement between the different modelling strategies. Wearing et al. (2000) analysed the model of Owen \& Sherratt (1998) further, performing an extended linear analysis to describe the patterning behaviour of the model and derived an approximation to the fastest-growing patterning modes (i.e. those which one might expect to observe in nonlinear simulations) for different regions of parameter space. However, the linear predictions failed to capture the qualitative behaviour of the nonlinear system. Motivated by this, Wearing \& Sherratt (2001) performed a nonlinear analysis of a two-cell system, together with bifurcation analysis to gain insights into the behaviour of larger systems, highlighting that linear analysis alone is unable to predict the model's behaviour. Furthermore, this study concluded that the fine-grained patterns with approximate periodicity one sees in early development are due to the patterning dynamics rather than environmental inhomogeneity. Webb \& Owen (2004) extended this signalling model, considering lateral induction and inhibition (up- and down-regulation of ligand or receptor production in response to binding) in systems of varying geometry; specifically, one-dimensional strings and arrays of square or hexagonal cells were considered. Via linear analysis, the fastest growing modes were again calculated explicitly.

In this paper, we extend the work of Wearing et al. (2000) and Wearing \& Sherratt (2001) to present a thorough investigation of the pattern-forming behaviour of the model of Owen \& Sherratt (1998) in which ligand molecules, unoccupied receptors and bound ligand-receptor complexes are modelled. Lateral induction and lateral inhibition are considered so that ligand production may either increase or decrease with the current level of occupied receptors. We present a linear stability and bifurcation analysis of the model equations, as well as numerical simulations, to both illustrate and to provide new insight into the failure of the linear analysis to capture the patterning behaviour of the nonlinear system. Our analysis also highlights the variety of stable patterns that exists at a given choice of parameter values associated with feedback strength; additionally, by analysing the results of numer- 
ical simulations run to steady state (from a variety of initial signalling profiles) in both the spatial and frequency domains, the dominant pattern for a given parameter choice is discerned. Finally, by sampling patterns of non-integer wavelength on a discrete mesh, we show how the continuous and discrete representations of such signalling mechanisms may differ: in the continuous case, patterns of arbitrary wavelength may be generated which do not necessarily fit onto a discrete lattice, while in the discrete case such patterns result in longer wavelength harmonics being selected where the wavelength is rational; in the case of initial conditions of irrational pattern wavelength, the resulting pattern is found to be aperiodic.

The remainder of the paper is organised as follows. In $\S 2.1$, the model of Owen \& Sherratt (1998) is recapitulated; the linear stability properties presented in Wearing et al. (2000) are summarised in $\S 2.2$. In $\S 3$ bifurcation diagrams and numerical simulations, together with spatial and spectral analyses, are presented to illustrate the emergence and dominance of different patterning modes in parameter space. In $\S 4$, the evolution of patterns of non-integer wavelength is considered, showing how the continuous and discrete representations of such systems may deviate. In $\S 5$, a discussion of our results is given together with directions for future research.

\section{A mathematical model of juxtacrine cell signalling with feedback}

\subsection{Formulation}

In Owen \& Sherratt (1998), a model of juxtacrine cell signalling is presented. Such signalling is known to be of importance within closely packed cell populations, allowing membrane-bound signalling molecules to bind to receptors in adjacent cell membranes.

The mathematical model comprises ODEs which describe ligand-receptor binding on each cell and is expressed in terms of the numbers of ligand molecules $a_{j}(t)$, unoccupied receptors $f_{j}(t)$ and bound receptor-ligand complexes $b_{j}(t)$ on each cell $j$. A generic model is employed to represent ligand binding in which it is assumed that a single ligand molecule binds reversibly to a receptor on the cell surface, giving rise to an occupied receptor which is subsequently internalised within the cell. In practice new ligand and receptors are produced via recycling, release from intracellular stores, and de novo production; however, in Owen \& Sherratt (1998), the simplifying assumption was made that the cell's ligand and receptor production depends upon the level of occupied receptors in a prescribed way. By varying parameters associated with the feedback between ligand or receptor production and the level of bound receptors on a cell, up- or down-regulation of ligand and receptor production (known as lateral induction and inhibition) may be modelled.

The equations governing the evolution of ligand and free and bound receptors on each cell $j$ are, respectively (Owen \& Sherratt, 1998):

$$
\begin{aligned}
\dot{a}_{j} & =-k_{a} a_{j}\left\langle f_{j}\right\rangle+k_{d}\left\langle b_{j}\right\rangle-d_{a} a_{j}+P_{a}\left(b_{j}\right), \\
\dot{f}_{j} & =-k_{a}\left\langle a_{j}\right\rangle f_{j}+k_{d} b_{j}-d_{f} f_{j}+P_{f}\left(b_{j}\right), \\
\dot{b}_{j} & =k_{a}\left\langle a_{j}\right\rangle f_{j}-\left(k_{d}+k_{i}\right) b_{j},
\end{aligned}
$$

wherein dots denote differentiation with respect to time, $k_{a}$ and $k_{d}$ represent the 
rates of ligand binding and dissociation, $k_{i}$ the rate of ligand-receptor complex internalisation and $d_{a}$ and $d_{f}$ are the rates of decay of ligand and free receptors. The average over neighbouring cells is denoted $\langle\cdot\rangle$ and defined by

$$
\left\langle\psi_{j}\right\rangle=\frac{\psi_{j-1}+2 \psi_{j}+\psi_{j+1}}{4}
$$

corresponding to an average over a two-dimensional array of square cells with variation in one dimension only. The functions $P_{a}\left(b_{j}\right), P_{f}\left(b_{j}\right)$ represent the production of ligand and free receptors and are specified as follows:

$$
P_{a}(x)=\frac{C_{1}^{m} x^{m}}{C_{2}^{m}+x^{m}}, \quad P_{f}(x)=C_{3}+\frac{C_{4}^{n} x^{n}}{C_{5}^{n}+x^{n}},
$$

so that lateral inhibition or induction is captured by appropriate choice of the parameters $C_{1}-C_{5}$ and the exponents $m, n$ and was exploited in Wearing \& Sherratt (2001); e.g. $C_{2}^{m}<0$ leads to inhibition of ligand production in response to ligandreceptor binding.

The data available on production rates of ligand and receptors are typically extremely limited; however, the parameters in (5) may be specified to some extent, as follows (see Owen \& Sherratt (1998)). Equilibrium levels of free and bound receptors are frequently known for specific biological systems; defining background receptor expression $r_{0}$ and spatially-homogeneous steady-states $\left(f^{*}, b^{*}\right)$ specifies the remaining steady-state, $a^{*}$, and three relations between the parameters in (5), leaving four parameters unspecified $C_{2}, m$ and $C_{5}, n$, which reflect the strength of feedback in ligand and free receptor production, respectively. We choose $C_{2}$ and $C_{5}$ as free parameters with which to investigate the model's patterning behaviour in regimes corresponding to both lateral induction and inhibition, and fix the exponents to take the values $n=m=3$; similar behaviour may be obtained by varying the exponents $m$ and $n$. In vivo, ligand-receptor binding and the resulting ligand expression are dependent on the cell's biochemical and biophysical environment; experimental evidence suggests that such environmental inhomogeneities are significant, providing motivation for the consideration the range of patterns produced by this model under variation of the feedback parameters.

\subsection{Linear stability analysis}

By expanding around the homogeneous steady states Owen \& Sherratt (1998) and Wearing et al. (2000) showed that the values of $P_{a}^{\prime}\left(b^{*}\right), P_{f}^{\prime}\left(b^{*}\right)$ (here denoted $\mathscr{A}, \mathscr{F}$, respectively) dictate the behaviour of solutions; varying the parameters $C_{2}$ and $C_{5}$ allows us to proceed in $\mathscr{A}-\mathscr{F}$ space to obtain different model behaviour. Curves separating different patterning solutions were defined in the $\mathscr{A}-\mathscr{F}$ plane as follows (see Wearing et al. (2000) for details).

Linearising about the steady state via $\left(a_{j}, f_{j}, b_{j}\right)=\left(a^{*}, f^{*}, b^{*}\right)+\delta\left(a_{1 j}, f_{1 j}, b_{1 j}\right)$ and seeking solutions of the form $a_{1 j}=\bar{a} e^{\sigma t+i k j}$ (where $\bar{a}$ is constant, $\sigma$ is the temporal growth rate and $k$ is the wavenumber), yields a cubic dispersion relation:

$$
\sigma^{3}+\alpha_{1} \sigma^{2}+\alpha_{2}(\mathscr{K}) \sigma+\alpha_{3}(\mathscr{K})=0
$$

wherein $\alpha_{1}, \alpha_{2}$ and $\alpha_{3}$ are functions (omitted; see equation (6), Wearing et al. (2000)) of the model parameters $k_{a}, k_{d}, k_{i}, d_{a}, d_{f}$, of the steady states $a^{*}, f^{*}, b^{*}$, 
of the gradients $\mathscr{A}, \mathscr{F}$ and (except for $\alpha_{1}$ ) of the wavenumber $k$, via:

$$
\mathscr{K}(k)=\frac{\cos (k)+1}{2} .
$$

Contours of neutral stability and of maximal instability in $\mathscr{A}-\mathscr{F}$ space for each wavenumber may easily be obtained from equation (6), indicating the regions of feedback parameter space in which the homogeneous state becomes linearly unstable to different pattern wavelengths. Maximal instability contours for a similar system are presented in Webb \& Owen (2004) and an approximation to those corresponding to equation (6) in Wearing et al. (2000).

The regions of parameter space in which linear analysis predicts spatial pattering are determined by analysis of the dispersion relation (6). This analysis was previously presented in Owen \& Sherratt (1998) and Wearing et al. (2000) so we omit the details. Referring to Figure 1, the results are summarised as follows:

1. Stability to homogeneous perturbations demands that the roots of (6) have negative real part, requiring $\alpha_{1}>0, \alpha_{3}(1)>0$ and $\alpha_{1} \alpha_{2}(1)-\alpha_{3}(1)>0$. These conditions define two straight lines in the $\mathscr{A}-\mathscr{F}$ plane (denoted $\mathscr{L}_{1}$ and $\mathscr{L}_{2}$, respectively) delimiting the stable region.

2. Instability to inhomogeneous perturbations requires at least one positive root of $(6)$, corresponding to $\alpha_{1} \alpha_{2}(\mathscr{K})<0$ or $\alpha_{3}(\mathscr{K})<0$. These conditions yield a straight line $\mathscr{L}_{3}$ as well as a curve, $\mathscr{C}$ which bounds the region in which complex roots are obtained. A further line $\mathscr{L}_{4}$ divides the stable region into two parts. This line bounds the region in which the smallest root of $\alpha_{3}(\mathscr{K})$ is zero.

3. The wavelength of the fastest growing modes in region II is bounded along the line $\mathscr{L}_{4}$, above which $\mathscr{K} \in\left[0, \frac{1}{2}\right]$; i.e. the fastest growing wavelength is $\lambda \in[2,4)$. Wavelengths of two or three cells are therefore expected to dominate the pattern form; no such restriction is found for values of $\mathscr{F}$ lying below the line $\mathscr{L}_{4}$.

Figure 1(a) shows a sketch of the $\mathscr{A}-\mathscr{F}$ plane illustrating the regions defined by the lines $\mathscr{L}_{1}-\mathscr{L}_{4}$ and the curve $\mathscr{C}$. Figure $1($ b) shows a numerically calculated version of this stability diagram with neutral stability contours superimposed (calculated from equation (6)). Below each of these contours, the homogeneous steady state is linearly stable to periodic perturbations of that period; above, the linear analysis predicts patterns forming. Figure 1(c) shows contours of the fastest growing patterning modes. We remark that the neutral stability contours shown in Figure $1(\mathrm{c})$, which indicate that longer range patterns are expected to emerge as the rate of free receptor production increases, are also consistent with the linear analysis of Wearing et al. (2000), in which it is predicted that patterns of period 2 or 3 will dominate in region II. By considering an alternative form for the nearest neighbour interaction (4), Webb \& Owen (2004) obtained qualitatively different behaviour to that shown in Figure 1(c); however, by suitable re-definition of $\mathscr{K}$, we may reproduce their results (omitted). 


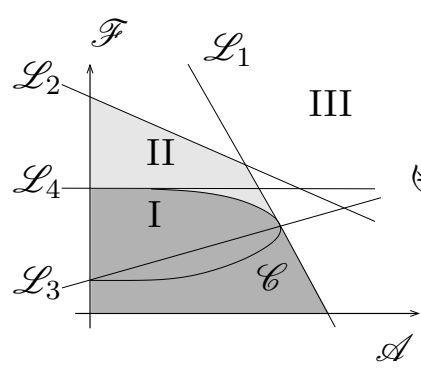

(a)

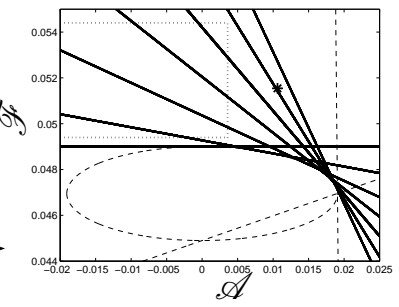

(b)

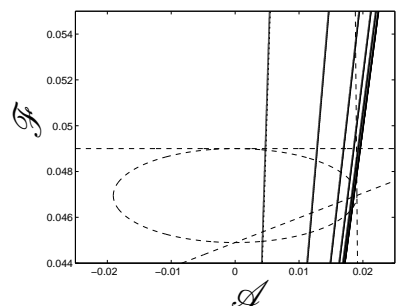

(c)

Figure 1: (a) A sketch of the $\mathscr{A}-\mathscr{F}$ plane denoting the regions of stable homogeneous solutions (I), stable periodic patterns (II) and instability of the homogeneous steady state to homogeneous perturbations (III). Adapted from Wearing et al. (2000); (b) neutral stability contours in the $\mathscr{A}-\mathscr{F}$ plane for patterns of increasing period $\lambda=2-8$ (solid lines: period increases clockwise) and (c) contours of fastest-growing patterning modes $\lambda=2-7$ (solid lines: period increases clockwise) together with the curves $\mathscr{L}_{1}, \mathscr{L}_{3}, \mathscr{L}_{4}$ and $\mathscr{C}$ delimiting the regions in parameter space in which patterns may be formed (dashed lines). Parameter values taken from Owen \& Sherratt (1998): $k_{a}=0.0003 \mathrm{molecules}^{-1} \mathrm{~min}^{-1}, k_{d}=0.12 \mathrm{~min}^{-1}, k_{i}=0.019 \mathrm{~min}^{-1}, d_{a}=0.006 \mathrm{~min}^{-1}$, $d_{f}=0.03 \mathrm{~min}^{-1}, f^{*}=b^{*}=3000$, except $r_{0}=2850, m=n=3$. The dotted box in (b) shows the region of parameter space investigated in more detail in $\S 3$; the asterisk indicates the parameter value employed in the numerical simulations shown in Figure 2.

\section{Numerical simulations and bifurcation analysis}

\subsection{Pattern emergence and stability}

The linear analysis of Wearing et al. (2000) and Webb \& Owen (2004) enables partitioning of parameter space into regions in which stable patterns are generated from the homogeneous steady state, and enables determination of the fastest-growing modes. However, the applicability of such a linear analysis depends on whether the bifurcation at which the pattern under consideration is created is of supercritical type; in the case of subcritical patterning bifurcations, the linear approximation fails to reflect the patterning dynamics. Indeed, numerical simulation of the nonlinear system reveals that the linear predictions fail (unsurprisingly) to capture even the qualitative patterning behaviour of the system. In the following section, we present numerical simulations, together with bifurcation diagrams, for parameter values corresponding to both lateral inhibition and induction to demonstrate the emergence of different regular patterning modes; furthermore, since the analysis of the fastest growing mode has proved a poor predictor of which patterns will dominate, we calculate via numerical simulation the dominant patterns for each parameter set for a range of initial data.

The system (1)-(3) is solved using the initial value problem solver ode15s in MATLAB. A typical numerical simulation for parameter values such that ligand and receptor production are both upregulated (lateral induction) and stable patterns exist is shown in Figure 2; such approximately periodic patterns are typical 
in the simulations performed and observed in early tissue development (Wearing \& Sherratt, 2001).
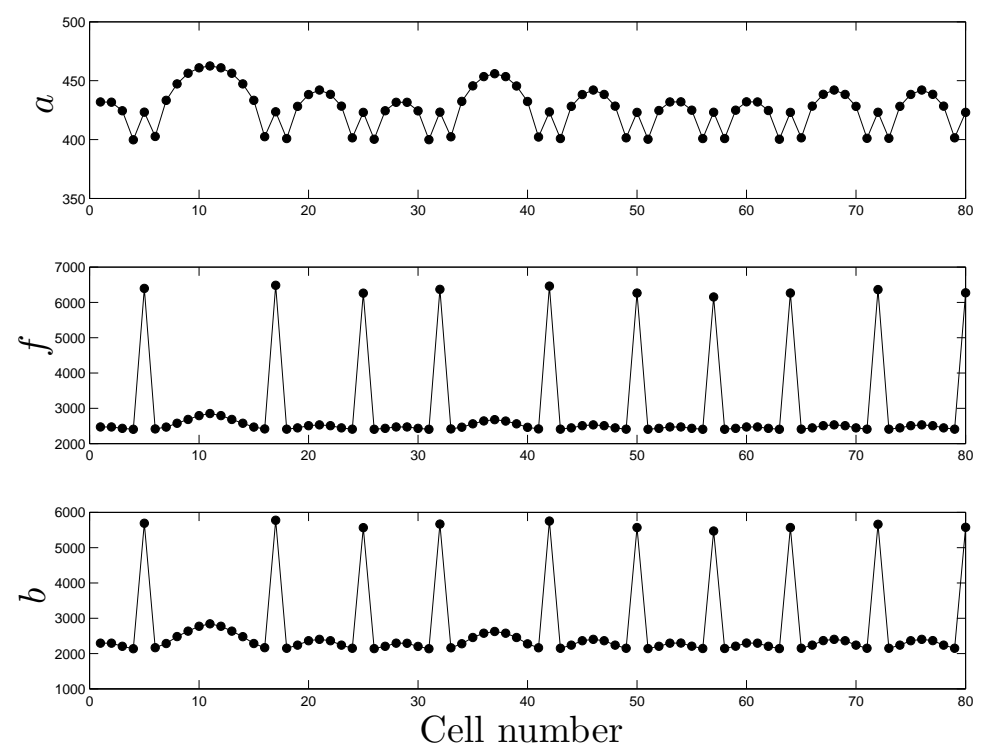

Figure 2: The steady-state pattern obtained from numerical simulation of the system (1)-(3) at $t=1000 \mathrm{~h}$ in a line of 80 cells with periodic boundary condition indicating that the patterns typically obtained from (1)-(3) display approximate periodicity with defects. Parameter values are as in Figure 1 with $C_{2}$ and $C_{5}$ chosen such that $\mathscr{A}, \mathscr{F}$ lie in region II (as indicated by the asterisk in Figure 1(b)), in which stable patterns are predicted by linear analysis. Initial conditions are random perturbations about the homogeneous steady states.

We now investigate the emergence of different regular pattering modes. The system may, in principle, be driven towards a mode of arbitrary integer period $\lambda$ by choosing suitable periodic or near-periodic initial conditions and domain size, for an appropriate parameter choice at which such a mode is stable. We remark that tacit in the below is the assumption that only one such patterning mode exists (up to cyclic permutation); our numerical investigations suggest this to be the case. Figure 3 depicts sample bifurcation diagrams (together with sample simulation results) for the system (1)-(3). Figures $3(\mathrm{a})$ and (c) illustrate the stability of the $\lambda=3$ and $\lambda=4$ patterns as the free receptor production parameter $C_{5}$ is varied in the lateral induction regime. In each case the solution branches indicate the number of free ligand molecules on each cell in the periodic unit; the homogeneous state is indicated by the horizontal solution curve, and the patterned states by the remaining branches. For example, in the period three regime (Figure 3(a)), the number of ligand molecules on two of the three cells follows the upper solution branch, the remaining cell tracking the lower branch. Similarly, in the period four regime, one cell tracks each of the upper and lower branches, the remaining two cells following the intermediate branch. Longer wavelength patterns display qualitatively similar behaviour; however, the behaviour becomes highly complex for larger patterns, with many solution modes coexisting (see Wearing \& Sherratt (2001) in which bifurcation 
diagrams on 8 cells are shown).

Figures 3(a) and (c) give an insight into why the linear analysis fails to give a complete description of the patterning behaviour of the system (1)-(3). Patterns may be formed in regions of parameter space below the relevant neutral stability curve predicted by the linear analysis (shown in Figure 1(b)) since the solution branches can fold subcritically from the homogeneous steady-state to form stable patterning regimes in regions of parameter space where the homogeneous state is linearly stable to periodic perturbations. An alternative mechanism for this phenomena is that the non-fastest-growing patterning mode is stable as a nonlinear pattern. We emphasise that since the uniform state is linearly stable to non-uniform perturbations, the competition between states that we are describing should be viewed in terms of bistability, rather than of a Turing pattern, for example, arising through propagation over an unstable state.

We pause here to note that, not only do the linear instability curves shown in Figure 1(b) fail to represent the emergence of patterns in the nonlinear system, but the fastest-growing modes shown in Figure 1(c) (often good predictors of observed patterning behaviour) are similarly inaccurate.

The supercritical pitchfork bifurcation which branches from the homogeneous steady-state in Figure 3(c) represents the $\lambda=2$ bifurcation which occurs as the receptor production is increased across the line $\mathscr{L}_{4}$; see Figure 1(b) (separate plot omitted for concision). This supercritical bifurcation, which exists for a wide range of parameter values corresponding to lateral inhibition, is exploited in the Appendix, in which a multiscale analysis is employed to demonstrate that complex discrete pattern-forming models of this type may be accommodated within tissue-scale representations; subcritical bifurcations typically preclude useful application of such an analysis since the unstable solutions reflected in the multiscale asymptotic equations will not be observed in nonlinear simulations. For values of $C_{5} / b^{*}$ corresponding to $\mathscr{F}$ lying above the line $\mathscr{L}_{4}$ in the $\mathscr{A}-\mathscr{F}$ plane, the $\lambda=4$ pattern branches subcritically from the $\lambda=2$ solution. Figure 4 shows how the $\lambda=4$ pattern is generated from the $\lambda=2$ solution under variation of the ligand production parameter $C_{2}$ in the lateral inhibition regime: again, the $\lambda=4$ solution branches subcritically from the shorter period pattern.

\subsection{Dominant patterning modes}

As remarked above, linear analysis of the fastest growing modes proves a poor indicator of the pattern wavelengths generated by the full nonlinear system. We wish to determine the dominant pattern period for a given parameter choice; these were determined via numerical simulation as follows. Solutions to (1)-(3) were obtained, from a series of initial states consisting of a patch of stable pattern of period $\lambda$ (of width $10 \lambda$ cells) surrounded by the homogeneous state (so that a wide array of pattern wavelengths are admitted, 120 additional cells are used). The resulting steady-state solutions were analysed in both the frequency and the spatial domains.

Spectral analysis (or frequency domain analysis) provides a simple and powerful way to identify the (spatial) frequency components in patterned solutions and is widely used in signal processing applications (though in these applications, the data usually take the form of a time-series, rather than a spatial distribution; see, e.g., Broughton \& Bryan (2009)). The patterning energy at each spatial frequency 


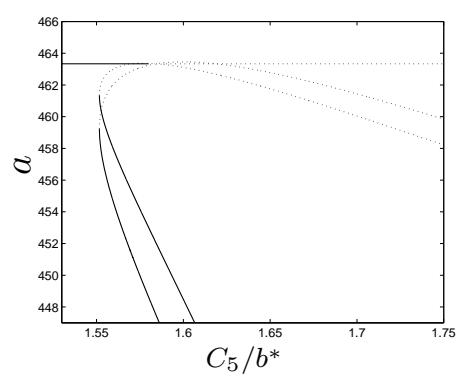

(a)

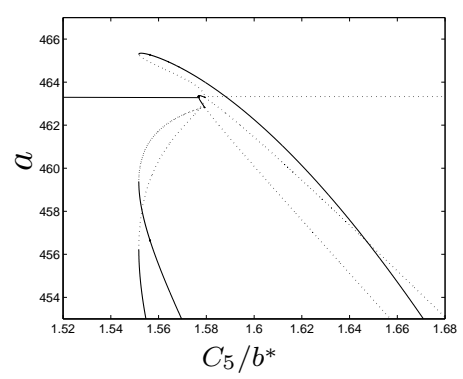

(c)

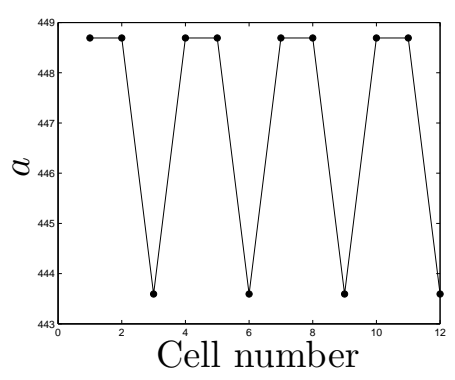

(b)

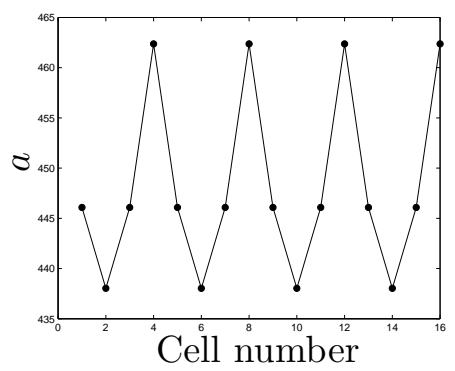

(d)

Figure 3: (a) and (c): Bifurcation diagrams illustrating the subcritical bifurcation of the $\lambda=3, \lambda=4$ patterns for the number of ligand molecules $a$ from the homogeneous state for system (1)-(3) (solutions for bound and free receptors are omitted for brevity); (b) and (d) show sample numerical simulation illustrating the corresponding patterns generated in a line of 120 cells with periodic boundary conditions and $t=1000 \mathrm{~h}$. Parameters as in Figure 1 except $C_{2}=1000$ and in, (b), (d), $C_{5}=1.59 b^{*}$. The supercritical pitchfork bifurcation which branches from the homogeneous steady-state in (c) represents the $\lambda=2$ pattern generation. 


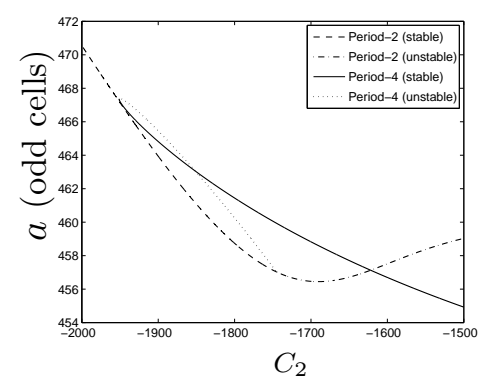

(a)

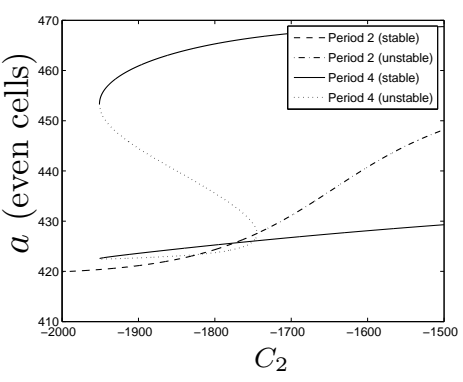

(b)

Figure 4: Bifurcation diagrams indicating how the period-4 pattern for ligand concentration branches from the period-two solution under variation of $C_{2}$ on (a) odd cells and (b) even cells. Stable and unstable period-two and four solutions are indicated by the different line styles. Parameters as in Figure 3 except $C_{5}=1.6 b^{*}$ and $C_{2}$ as indicated.

is measured by the power spectral density, calculated as the scaled absolute value of the square of the discrete Fourier transform of the steady-state solution. The power spectral density is scaled so that its mean and variance are equal; the discrete Fourier transform is obtained via the $\mathrm{ft}$ function in MATLAB. Energy peaks at distinct frequencies indicate the presence (and prevalence) of different patterning modes. In the spatial domain, we calculate separately the stable periodic patterns that exist at each parameter choice and compare numerically these against the steady-state solutions obtained from time-dependent simulations, thereby providing an explicit measure of the appearance of different periodic cycles in the domain. By these two approaches we may isolate the different patterns that exist in the domain and calculate the dominant patterning mode for each parameter set: we characterise the dominance by the percentage of the domain occupied by that pattern.

Figure 5(a) shows an illustrative steady state pattern in which both $\lambda=2$ and $\lambda=3$ patterning modes coexist in the domain; Figure 5(b) indicates the power spectrum associated with this pattern, indicating how such an analysis clearly highlights the patterning modes present via distinct peaks of power spectral density at $\lambda=2$ and $\lambda=3$. We remark further that it is relatively easy to construct patterned solutions whose spatial distribution appears similar but whose spectrum shows distinct differences. This exemplifies the value of the spectral analysis method and further motivates the complementary use of both spatial and frequency domain analysis.

Figure 6 shows a plot of the dominant patterns in a region of $\mathscr{A}-\mathscr{F}$ space (corresponding to the region of Figure 1(b) marked with a dotted box), together with its percentage dominance. For clarity, the axes are labelled with the values of the feedback parameters $C_{2}$ and $C_{5}$ since the corresponding non-uniform $\mathscr{A}-\mathscr{F}$ mesh obscures detail near $\mathscr{A}=0$. Figures 6(c) and (d) show the dominant patterns for two representative values of free receptor upregulation $\left(C_{5}=1.6 b^{*}, 1.95 b^{*}\right)$ together with their percentage dominance. Figure 7 shows the dominant patterns resulting from an initial state comprising a region of random, rather than periodic, data surrounded by the unstable homogeneous state. 


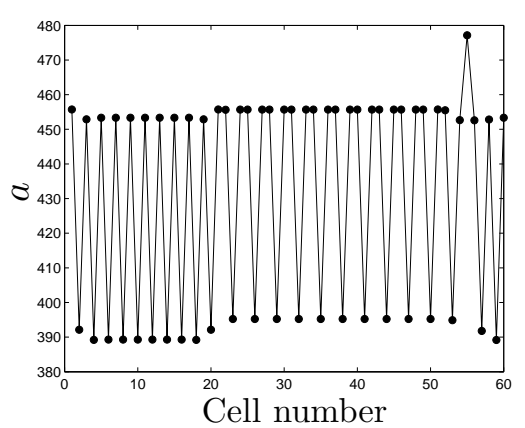

(a)

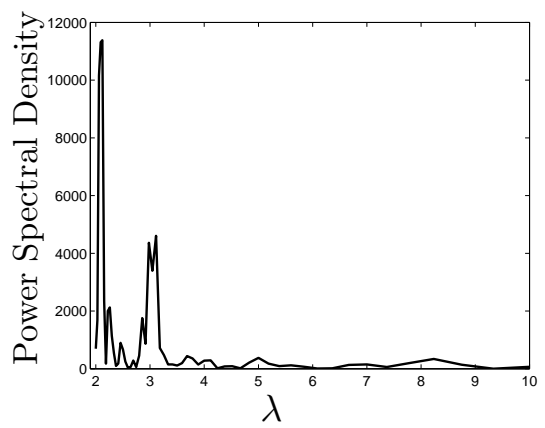

(b)

Figure 5: (a) A section of a numerical simulation run to steady state in a periodic line of 120 cells, in which $\lambda=2$ and $\lambda=3$ patterning modes are present. The parameter values are as in Figure 3 except $C_{5}=1.85 b^{*}, C_{2}=-1850$. (b) The power spectral density estimate corresponding to the simulation shown in (a).

Comparison of Figures 1(b,c) and 6(a) shows clearly that, due to the subcritical nature of the patterning bifurcations, patterns form in some regions of parameter space in which the homogeneous steady state is linearly stable to periodic perturbations. Furthermore, the fastest growing modes predicted by the linear analysis provide a poor description of the nonlinear behaviour, except for very strong ligand inhibition at which $\lambda=2$ patterns are predicted and observed. As the inhibition is reduced $\lambda=3$ and $\lambda=4$ solutions achieve dominance. A region of $\lambda=4$ dominance is observed between the $\lambda=2$ and $\lambda=3$ regions. Under induction, $\lambda=4$ regains dominance together with other, longer-wavelength patterns $(\lambda=5,6,9)$. Figures $6(\mathrm{~b}-\mathrm{d})$ indicate that the dominance of the patterns is greatly reduced for lateral induction $(\mathscr{A}>0)$, the resulting patterns containing many defects. Comparison of Figures 7 and $6(\mathrm{c}, \mathrm{d})$ indicates that the same patterning trends are observed in the case for which the initial state comprises random perturbations to the homogeneous steady state: at strong inhibition, short range patterns dominate; longer-range patterns dominate under induction. We note that, apart from the case of very strong inhibition (in which the alternating pattern is robustly generated), the dominance of these patterning modes is very greatly reduced, and the resulting steady state patterns display mixtures of stable patterning modes with many defects (figure omitted). Combined, these results further exemplify that, with the emergence of more stable patterning modes (as inhibition is decreased), the predictions of the linear analysis shown in Figure 1 comprehensively fail to capture the nonlinear behaviour.

We pause to remark that, though we are primarily concerned with the system's steady-state behaviour, the simulation dynamics provide many interesting features for investigation. The initial patch of stable pattern may invade the (unstable) homogeneous state via a travelling wave; alternatively, a travelling wave of a different mode may invade the homogeneous state and the original stable pattern. In some cases, propogation failure leads to pinned regions between the original and alternative, invading, pattern. Lastly, an apparently incoherent mix of (distorted) pattern modes may evolve. Characterisation of these dynamics in terms of the parameter 
values forms ongoing work, adding to existing investigations by, e.g. Owen (2002) .

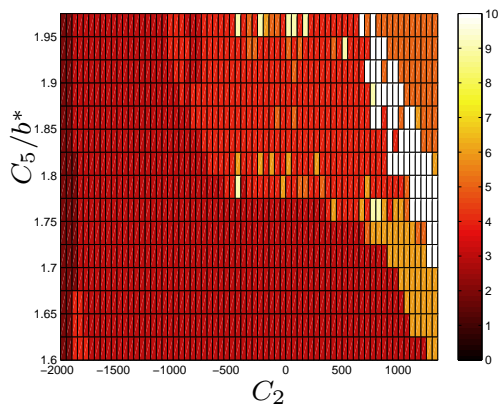

(a)

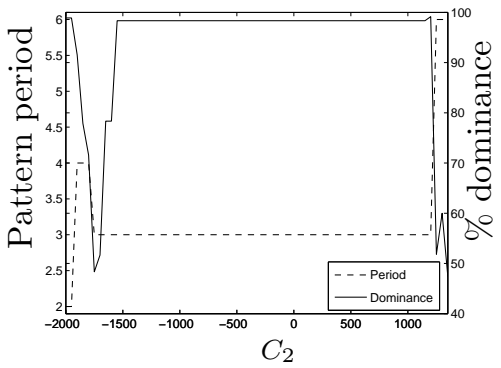

(c)

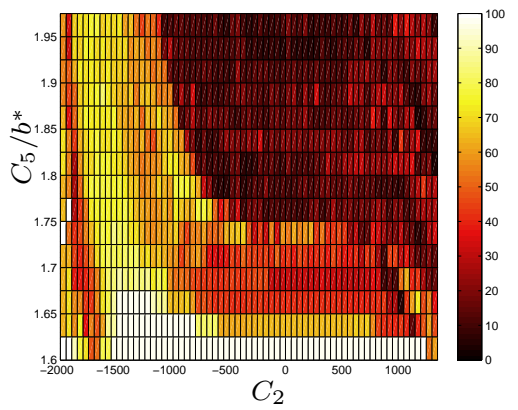

(b)

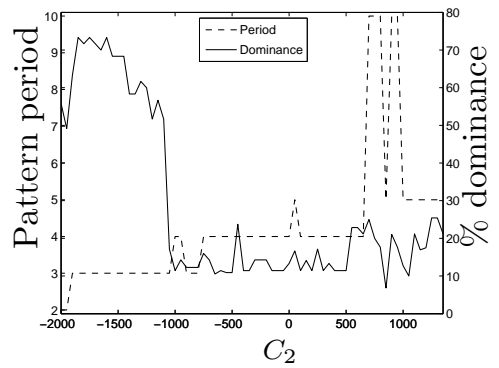

(d)

Figure 6: (a) The dominant pattern period in a region of $\mathscr{A}$ - $\mathscr{F}$ space, (b) the percentage of the domain occupied by that pattern as a measure of its dominance, and the dominant patterns for two representative values of free receptor upregulation together with their percentage dominance: (c) $C_{5}=1.6 b^{*}$, (d) $C_{5}=1.95 b^{*}$. Simulations undertaken in a line of $10 \lambda+120$ cells with periodic boundary conditions. Parameters as in Figure 3 except $C_{2}, C_{5}$ as indicated.

\section{Non-integer patterns}

The simulations presented in $\S 3$ demonstrate that many different stable patterns exist at each parameter value; furthermore, the patterns generated from the nonlinear model do not adhere to the linear predictions presented in $\S 2.2$. In addition, the resulting patterns generally contain defects. The linear analysis applies for both continuous and discrete systems; as such, the wavenumber, $k$, is arbitrary and may correspond to non-integer pattern wavelength. In the discrete case, this leads to patterns which do not fit onto the lattice. Below, we investigate the behaviour of such patterns, highlighting a point at which the equivalent discrete and continuum representations of signalling phenomena diverge.

We demonstrate the behaviour of non-integer wavelength patterns in our discrete framework by simulating the system (1)-(3) with initial conditions comprising a 


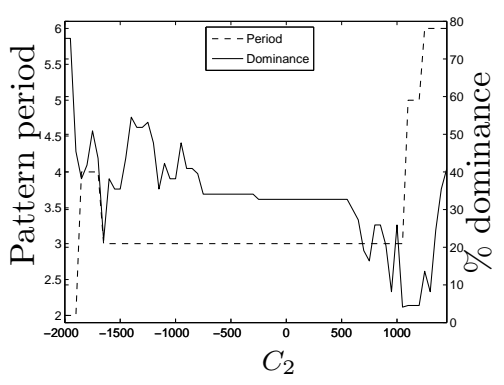

(a)

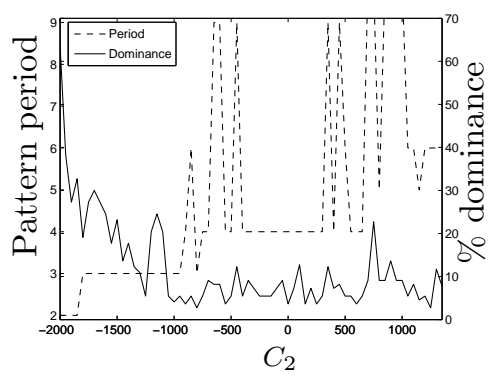

(b)

Figure 7: Diagrams showing the dominant pattern, together with its percentage dominance resulting from an initial state comprising a region of random data surrounded by the unstable homogeneous steady state for for two representative values of free receptor upregulation: (a) $C_{5}=1.6 b^{*}$, (b) $C_{5}=1.95 b^{*}$. Other parameters as in Figure 3 except $C_{2}$ as indicated.

pattern of non-integer period sampled on the discrete mesh. Figure 8 shows initial conditions of period $\lambda=2.8$ together with the resulting steady-state pattern.

Figure $8(\mathrm{a})$ illustrates how sampling patterns of rational non-integer wavelength $\lambda$ (with wavenumber $k$ ) on a discrete mesh leads to patterns of longer wavelength $\bar{\lambda}$ (with wavenumber $\bar{k}$ ) given by:

$$
\bar{\lambda}=\frac{2}{\bar{k}} ; \bar{k}=\min \{n k(\bmod 2), 2-n k(\bmod 2)\} ; \bar{\lambda}, n \in \mathbb{Z} .
$$

Equivalently, $\bar{\lambda}$ may be interpreted as the shortest integer multiple of the original pattern that fits onto a discrete mesh; i.e.

$$
\bar{\lambda}=\min \{m \lambda\} ; \text { where } m \in \mathbb{Z} \text { satisfies } m \lambda(\bmod 2)=0 .
$$

Returning to the case for which $\lambda=2.8$, we find $\bar{\lambda}=14(n=50, m=6)$; inspection of Figure 8 readily confirms this. Such wavelength selection 'errors' relate to aliasing effects observed in signal processing applications, in which the sampling frequency can cause signals of disparate wavelength to become indistinguishable.

We remark that Figure 8 (b) indicates that the steady-state pattern of period $\bar{\lambda}=14$ can, roughly speaking, be thought of as being constructed from (distorted) patterns of shorter wavelength (here, patterns of period $\lambda=5$ and $\lambda=3$ ). We note further that a straightforward linear analysis reveals that the homogeneous steady-state is stable to period-14 perturbations in the chosen parameter regime. We therefore conclude that the $\lambda=14$ pattern bifurcates subcritically from the homogeneous steady-state in a similar manner to that shown in Figures 3(c) and 4 (we do not present a corresponding bifurcation diagram since the behaviour is exceedingly complex, with many solution branches).

For patterning modes of irrational period, Equations (8), (9) of course predict that there is no pattern that fits on the discrete lattice. For initial conditions of irrational period (in practice meaning that they do not correspond within numerical error to a rational that would lead under (8), (9) to a period observable within the 
number of grid points used), numerical simulation suggests that the period of the resulting pattern becomes very large. For example, Equations (8), (9) predict patterns of wavelength $\bar{\lambda}=42426$ from initial data of period $\lambda=2 \sqrt{2}$, truncated to 4 decimal places. However, we remark that the resulting pattern is 'locally periodic', consisting of smaller stable patterns that are stable at that choice of parameter value, as was demonstrated for the $\lambda=14$ pattern and shown in $8(\mathrm{~b})$. Figure 9 shows the pattern resulting from initial conditions of $\lambda=2 \sqrt{2}$ (truncated to 4 decimal places). The simulation was undertaken on an array of $3 \bar{\lambda}$ cells. The resulting distribution of free ligand appears to display short-range periodicity; however, small defects in the pattern mean that the true period, $\bar{\lambda}$, is very much larger. This highlights a disparity which can arise between the discrete and continuum representations of the same patterning system: in the continuum case, period-selection issues do not arise and the wavenumber $k$ is arbitrary; however, in the discrete model, initial data of fractional (or irrational) period can result in the system evolving to surprisingly long-wavelength patterns. These patterns comprise smaller patterns that are stable at that choice of parameter value, with defects. These 'locally periodic' patterns are consistent with the patterns one observes experimentally; Wearing \& Sherratt (2001) also observed such approximately periodic patterns, concluding that these are due to the patterning dynamics rather than environmental heterogeneity. The results presented in this section suggest that being driven towards a non-integer patterning state (via initial conditions and feedback characteristics) provides a simple explanation for the emergence of such patterns.

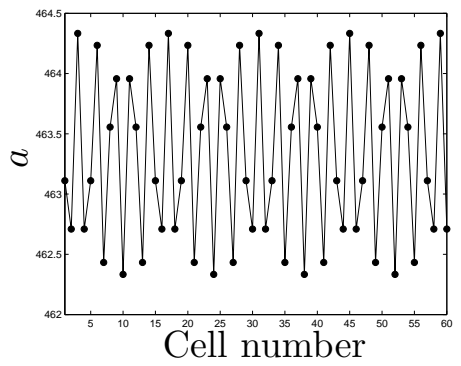

(a)

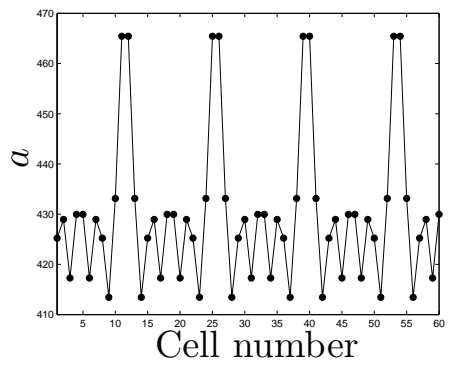

(b)

Figure 8: (a) Initial conditions corresponding to period $\lambda=2.8$ demonstrating the $\bar{\lambda}=14$ periodicity on the discrete lattice, and (b) the eventual $\bar{\lambda}=14$ pattern comprising distorted $\lambda=5$ and $\lambda=3$ patterns. $C_{2}=0, C_{5} / b^{*}=2.8$; other parameters as in Figure 2.

\section{Discussion}

In this paper, we have presented an investigation into the patterning behaviour of a discrete model of juxtacrine cell signalling due to Owen \& Sherratt (1998). The model comprises ODEs describing ligand-receptor binding activity on discrete cells. Three populations are considered: ligand molecules, unoccupied receptors and bound ligand-receptor complexes. Feedback between the number of bound receptors 


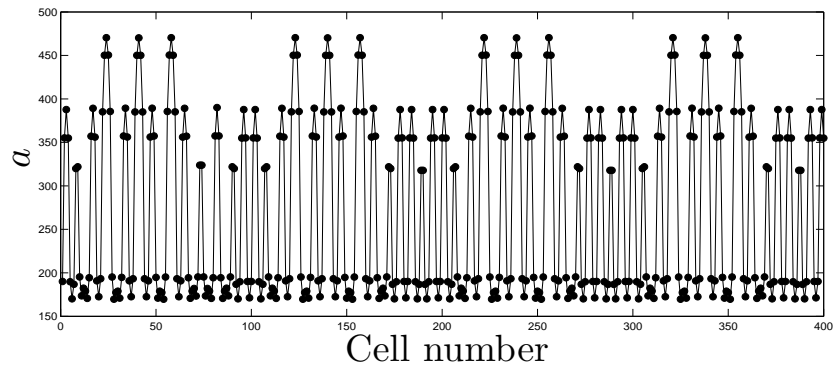

Figure 9: The steady-state ligand pattern resulting from an initial state with period $\lambda=2 \sqrt{2}$ (truncated to 4 decimal places). $C_{2}=0, C_{5}=2.8 / b^{*}$; other parameters as in Figure 2.

on a cell and its production of new receptors and ligand was incorporated and, by varying two free parameters controlling the strength of this feedback, up- and downregulation of receptor production (known as lateral inhibition or induction) were modelled.

Via a linear analysis, bifurcation analysis and numerical simulation, the variety of stable patterns which exist, for different feedback parameters was characterised. We demonstrated that the majority of bifurcations which generate different patterning modes are subcritical (a supercritical pitchfork may be obtained in the period two case for certain parameter choices) and stable patterns may therefore be formed in regions of parameter space where the homogeneous state is stable to such periodic perturbations. This sheds light on the inability of the linear analysis to reflect the observed behaviour of the nonlinear system, as reported in Wearing et al. (2000), Wearing \& Sherratt (2001) and Webb \& Owen (2004) (and remarked in Plahte (2001) with regards to a simpler signalling model): at a given point in parameter space, a wide variety of stable patterns of disparate wavelengths exist overriding the wavelengths predicted by the linear analysis; indeed, we demonstrate that the fastest-growing linearly unstable modes (often good predictors of nonlinear patterning behaviour) are inaccurate. By analysing the results of numerical simulations in the spatial and frequency domains, the dominant pattern in a representative region of parameter space was determined, showing that shorter wavelengths dominate in the regime of lateral inhibition (and that the linear analysis is more successful at predicting the patterning behaviour in this regime), giving way to longer wavelength patterns with substantial defects under lateral induction.

The linear analysis presented herein may be applied to both continuous and discrete systems and the stability of patterns of arbitrary period may be discerned. Within a discrete framework, patterns of non-integer period do not have a clear meaning. We have demonstrated that sampling such (rational) non-integer patterns on a discrete lattice leads to the generation of longer wavelength harmonics; namely, the shortest integer multiple of the original pattern which fits onto the lattice. If patterns of this wavelength are unstable in that region of parameter space, shorter wavelength stable patterns are used to create it. If the wavelength under consideration is irrational, it will not be periodic on the lattice over any domain size and aperiodic solutions are obtained. By simulation we showed that for approximately irrational initial patterning modes the solutions obtained have very large period; 
however, they display 'local periodicity', being constructed from stable, shorterwavelength patterns with defects. This highlights a disparity between the discrete and continuum representations of cell signalling systems. In the continuum case, such period-selection behaviour is not observed and patterns of arbitrary wavelength may be obtained, and, in general, nonlinearities will generate higher harmonics. Furthermore, other studies of this system (Wearing \& Sherratt, 2001) have noted that the patterns created display, in general, short-range periodicity with many defects and that such patterns are consistent with those observed experimentally. Our analysis of non-integer patterns indicates that such patterns emerge naturally when the system is driven towards a non-integer patterning mode via initial conditions and feedback characteristics.

Our results illustrate the following more general issues in analysing discrete patterning systems. We followed the usual procedure of performing a linear analysis in order to partition parameter space into regions in which different patterning modes might be expected to exist. However, given that many of the bifurcations in question are (as we have highlighted) subcritical, such a linear analysis does not provide an accurate description of the nonlinear behaviour; a thorough bifurcation analysis is thus required to indicate patterning behaviour. It is worth noting that the linear analysis will be similarly ineffective if many (possibly generated at supercritical bifurcations) stable patterns exist for a given parameter set. For systems of this type, the a priori linear analysis is insufficient and a numerical analysis of the type employed herein is required. Numerical simulation of the model equations from a range of initial states together with a spatial- and/or frequency-domain analysis of the resulting steady-state patterns seem to represent the most effective ways to characterise the patterning behaviour.

The linear analysis summarised in this paper employs standard techniques. Wearing et al. (2000) noted that it is the specific form of the juxtacrine averaging term employed in this model (which corresponds to striped patterns in a square array) which distinguishes this patterning mechanism from Turing models, and that a term corresponding to discretised diffusion does not make sense biologically. However, it is markworthy that O'Dea \& King (2011a,b) employed a homogenisation technique to a simpler model of Delta-Notch signalling (Collier et al. (1996)) to show that, on the tissue scale, the juxtacrine interaction does indeed manifest itself as linear diffusion, at least in the short-range patterning regime. In the Appendix, we show that the technique is applicable to more complex signalling models, paving the way for their incorporation into tissue-scale studies, by applying it to Equations (1)-(3). We further remark that this technique may only be usefully applied to patterns formed at supercritical bifurcations; the predominance of subcritical bifurcations in Equations (1)-(3) has, therefore, implications for the application of such methods to cell signalling models.

In this paper, we have attempted to characterise the range of patterns produced by a specific cell signalling model. The model chosen is of fairly generic type and, for simplicity, we have considered striped patterns only. Interesting avenues of investigation include the influence of cell shape on the resulting dominant patterns, extending the work of Webb \& Owen (2004), and the characterisation of patterning dynamics in terms of the parameter values in such signalling systems. 


\section{Acknowledgements}

The authors gratefully acknowledge funding from BBSRC and EPSRC (BB/D008522/1). JRK also acknowledges the support of the Royal Society and Wolfson Foundation. All bifurcation diagrams were generated in XPPAUT v5.91.

\section{A A multiscale analysis of period-two patterns}

In this appendix, we demonstrate that, in the period-two patterning regime, the discrete juxtacrine signalling mechanism manifests itself as linear diffusion on the macroscale. The method employed is that presented by O'Dea \& King (2011a,b); the details are therefore omitted for brevity.

The results presented in this paper suggest that introducing appropriate spatial variation in the feedback parameters results in different patterning behaviour in certain regions of the domain. In a specific biological context, such parameter variation would correspond to differences in the sensitivity of the cells to Delta-Notch binding, leading to the adoption of a particular programme of gene activation by a subset of the cell population according to spatial position.

To construct such a continuum model, a homogenisation process is required. We assume that the spatial variation of the chosen parameter is slow compared to the variation of ligand and free and bound receptor numbers, thereby preserving the local periodicity in the patterning regime; that is, we construct a two-scale model. Biologically, this corresponds to analysis of microscale pattern formation in response to macroscale variation in cell-signalling activity (e.g. that induced by tissue-level chemical or physical stimulation). Considering a line of cells, we denote the distance between cells by $\delta \ll 1$ (see Figure 10), introduce a slowly-varying continuum variable $X=\delta j$ and slow timescale $T=\delta^{c} t$ (where $c>0$ is as yet unspecified) and represent the numbers of ligand, free and bound receptors in the multiple-scales form $a_{j}=a(j, X, T), f_{j}=f(j, X, T)$ and $b_{j}=b(j, X, T)$, in which $j$ and $X$ represent the rapidly-varying and the slow spatial scales, respectively. Additionally, since in the period-two patterning regime, neighbouring cells are expected to differ significantly in the numbers of ligand and free/bound receptors, we introduce the following notation to differentiate between "odd" and "even" cells:

$$
\begin{array}{ll}
\psi(j ; X, T)=\psi^{+}(X, T) & j=2 i+1 \\
\psi(j ; X, T)=\psi^{-}(X, T) & j=2 i
\end{array}
$$

where $\psi$ represents the numbers of ligand, bound and free receptors $(a, f, b)$ and $i$ is an integer. Exploiting this notation and on expanding in Taylor series, the spatial coupling term $\left\langle\psi_{j}\right\rangle$ defined by equation (4) may be written:

$$
\left\langle\psi^{ \pm}\right\rangle=\frac{1}{2}\left(\psi^{ \pm}(X, T)+\psi^{\mp}(X, T)+\frac{\delta^{2}}{2} \frac{\partial^{2}}{\partial x^{2}} \psi^{\mp}(X, T)+\mathcal{O}\left(\delta^{4}\right)\right) .
$$

In the following we derive coupled equations governing receptor and ligand activity in odd and even cells separately, facilitating the inclusion of fine-grained patterning phenomena within our continuum model.

The variation in feedback strength due to biochemical or biophysical conditions is for definiteness modelled by introducing a slow spatial variation to the parameters 


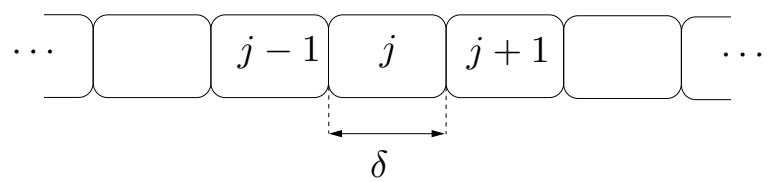

$X=\delta j$

Figure 10: Definition sketch: a line of cells and the spatial coordinates employed in the multiscale analysis.

$C_{2}$ and $C_{5}$, leading to variations in the gradient of the ligand and receptor production functions, which control the patterning behaviour. Figure 3 shows that (for suitable values of $C_{2}$ ) period-two solutions are created at a supercritical pitchfork bifurcation on the line $\mathscr{L}_{4}$ (see Figure 1(a)); correspondingly, we expand about $\mathscr{L}_{4}$ such that:

$$
\begin{aligned}
& P_{f}^{(k)}\left(b^{*}, X ; \delta\right)=P_{f}^{(k) *}+\delta^{2} F_{k}\left(b^{*}, X\right)+\cdots, \\
& \psi^{ \pm}(X, T ; \delta)=\psi^{*}+\delta \psi_{1}^{ \pm}(X, T)+\delta^{2} \psi_{2}^{ \pm}(X, T)+\cdots,
\end{aligned}
$$

wherein the superscript $(k)$ denotes the $k^{\text {th }}$ derivative of $P_{f}$ with respect to $b$ (a corresponding expansion is used for the ligand production rate $P_{a}$ ), and asterisks denote the values of the feedback functions (and their derivatives) at the bifurcation point on $\mathscr{L}_{4}$ at which $\psi$ achieves its homogeneous steady-state $\psi^{*}$. Exploiting the notation introduced in $\S 2.2$, we have $\mathscr{A}=P_{a}^{(1) *}+\delta^{2} A_{1}\left(b^{*}, X\right)$ and $\mathscr{F}=P_{f}^{(1) *}+$ $\delta^{2} F_{1}\left(b^{*}, X\right)$; the points $\left(P_{a}^{(1)^{*}}, P_{f}^{(1) *}\right)$ lie on the line $\mathscr{L}_{4}$. For parameter choices such that $F_{1}\left(b^{*}, X\right) \leqslant 0$, we therefore expect, as $t \rightarrow \infty$, spatially-homogeneous solutions to be approached; for $F_{1}\left(b^{*}, X\right)>0$, period-two solutions (generated at the supercritical pitchfork) exist.

For clarity, we note further that the supercritical bifurcation under consideration exists for a range of parameter values corresponding to lateral inhibition; the analysis below may only be usefully applied in this case.

Combining the above expansions and choosing $c=2$ (in order that both temporal and spatial coupling appears in the $\mathcal{O}(\delta)$ perturbation), and after some lengthy algebra, we obtain the following partial differential equation for the number of bound receptors on odd cells:

$$
\frac{\partial b_{1}^{+}}{\partial T}=\Lambda \frac{\partial^{2} b_{1}^{+}}{\partial X^{2}}+\mu b_{1}^{+3}+\nu b_{1}^{+} .
$$

The remaining variables are calculated via $f_{1}^{+}=\phi b_{1}^{+}$and $a_{1}^{+}=\chi b_{1}^{+}$and $\psi_{1}^{+}=-\psi_{1}^{-}$. The parameters $\Lambda, \mu, \nu, \phi, \psi$ and $\chi$ are cumbersome functions of the model parameters $\left(k_{a}, k_{d}, d_{a}, d_{f}, k_{i}\right)$, the steady-states $\left(a^{*}, f^{*}, b^{*}\right)$ and associated branch point feedback values and the perturbations to the feedback strength and are therefore omitted for brevity. We remark, however, that the signs of $\mu$ and $\nu$ dictate the patterning behaviour of the model. In particular, for constant $\mu, \nu$, the steady states of $(15)$ are $b_{1}^{+}=(0, \pm \sqrt{-\nu / \mu})$; for model parameter choices corresponding to $\mathscr{F}$ below $\mathscr{L}_{4}$, only the trivial state exists (corresponding to the maintenance of the homogeneous steady state); the change in sign of $\mu, \nu$ for parameter values at which $\mathscr{F}>\mathscr{L}_{4}$ leads to the generation of patterned states: $b \sim b^{*} \pm \delta \sqrt{-\nu / \mu}$. 


\section{References}

B. Appel, L.A. Givan and J.S. Eisen. 2001. Delta-Notch signaling and lateral inhibition in zebrafish spinal cord development. BMC Dev. Biol, 1:1-13.

S.A. Broughton and K. Bryan. 2009. Discrete Fourier analysis and wavelets: applications to signal and image processing. Wiley-Interscience. ISBN 0470294663.

J.R. Collier, N.A.M. Monk, P.K. Maini and J.H. Lewis. 1996. Pattern formation by lateral inhibition with feedback: a mathematical model of Delta-Notch intercellular signalling. J. Theor. Biol., 183:429-446.

A. Goriely, N. Dumont, C. Dambly-Chaudiere and A. Ghysen. 1991. The determination of sense organs in Drosophila: effect of the neurogenic mutations in the embryo. Dev., 113:1395.

V. Hartenstein and J.W. Posakony. 1990. A dual function of the notch gene in drosophila sensillum development. Dev. Biol., 142:13-30.

P. Heitzler and P. Simpson. 1991. The choice of cell fate in the epidermis of Drosophila. Cell, 64:1083-1092.

J.P. Keener and J. Sneyd. 1998. Mathematical physiology. Springer New York.

A.D. Lander, Q. Nie and F.Y.M. Wan. 2002. Do morphogen gradients arise by diffusion? Dev. Cell, 2:785-796.

T.A. Mitsiadis, K. Fried and C. Goridis. 1999. Reactivation of Delta-Notch signaling after injury: complementary expression patterns of ligand and receptor in dental pulp. Experimental Cell Res., 246:312-318.

C.B. Muratov and S.Y. Shvartsman. 2004. Signal propagation and failure in discrete autocrine relays. Phys. Rev. Lett., 93:118101(1-4).

R.D. O'Dea and J.R. King. 2011a. Continuum limits of pattern formation in hexagonal-cell monolayers. J. Math. Biol. DOI: 10.1007/s00285-011-0427-3.

R.D. O'Dea and J.R. King. 2011b. Multiscale analysis of pattern formation via intercellular signalling. Math. Biosci., 231:172-185.

M.R. Owen. 2002. Waves and propagation failure in discrete space models with nonlinear coupling and feedback. Phys. D: Nonlin. Phenomena, 173:59-76.

M.R. Owen and J.A. Sherratt. 1998. Mathematical modelling of juxtacrine cell signalling. Math. Biosci., 153:125-150.

M.R. Owen, J.A. Sherratt and S.R. Myers. 1999. How far can a juxtacrine signal travel? Proc. Royal Soc. B: Biol. Sci., 266:579-585.

K.J. Painter, P.K. Maini and H.G. Othmer. 1999. Stripe formation in juvenile Pomacanthus explained by a generalized Turing mechanism with chemotaxis. Proc. Nat. Acad. Sci. (US), 96:5549. 
E. Plahte. 2001. Pattern formation in discrete cell lattices. J. Math. Biol., 43(5): 411-445.

E. Plahte and L. Øyehaug. 2007. Pattern-generating travelling waves in a discrete multicellular system with lateral inhibition. Phys. D: Nonlin. Phenomena, 226 (2):117-128.

M. Pribyl, C.B. Muratov and S.Y. Shvartsman. 2003. Discrete models of autocrine cell communication in epithelial layers. Biophy. J., 84:3624-3635.

A.M. Turing. 1952. The Chemical Basis of Morphogenesis. Phil. Trans. Roy. Soc. Lond. Series B, Biol. Sci., 237:37-72.

S. Turner, J.A. Sherratt, K.J. Painter and N.J. Savill. 2004. From a discrete to a continuous model of biological cell movement. Phys. Rev. E, 69:21910/1-21910/10.

H.J. Wearing, M.R. Owen and J.A. Sherratt. 2000. Mathematical modelling of juxtacrine patterning. Bull. Math. Biol., 62:293-320.

H.J. Wearing and J.A. Sherratt. 2001. Nonlinear analysis of juxtacrine patterns. SIAM J. Appl. Math., 62:283-309.

S.D. Webb and M.R. Owen. 2004. Oscillations and patterns in spatially discrete models for developmental intercellular signalling. J. Math. Biol., 48:444-476.

L. Wolpert. 1969. Positional information and the spatial pattern of cellular differentiation. J. Theor. Biol., 25:1-47. 